\title{
Appel à contributions - Numéro spécial 2021 \\ Prévention et contrôle du tabagisme et du vapotage au Canada
}

Rédacteurs : Jennifer O’Loughlin (Université de Montréal), Thierry Gagné (University College London) et Robert Geneau (rédacteur en chef, Agence de la santé publique du Canada)

On estime que plus de 45000 Canadiens meurent chaque année d'une maladie liée au tabac ${ }^{1}$, ce qui fait du tabagisme la principale cause évitable de décès prématuré au Canada². De plus, la hausse récente d'utilisation de produits de vapotage, en particulier chez les jeunes, est une préoccupation croissante en santé publique. Des données probantes émergentes indiquent que les produits de vapotage ne sont pas sans risques pour les utilisateurs, mais des études supplémentaires sont nécessaires pour déterminer les risques à long terme. S'il n'est pas encadré par des règlements adéquats, le marché de la cigarette électronique pourrait se développer au point de devenir une menace à «l'endgame du tabagisme » ${ }^{3,4}$. Les politiques de contrôle du tabagisme et du vapotage sont désormais entremêlées.

Le Canada continue de mettre en œuvre des politiques et des programmes exhaustifs de contrôle du tabac dans le cadre de son engagement à atteindre un taux inférieur à $5 \%$ de tabagisme au pays d'ici $2035^{5}$. Au cours des dernières années, on y a également introduit des règlements sur les produits de vapotage au niveau fédéral ainsi que dans plusieurs provinces et territoires, avec l'objectif explicite de réduire l'utilisation de produits de vapotage chez les jeunes.

Le but de ce numéro spécial est de publier des données probantes scientifiques actuelles et émergentes sur l'épidémiologie, la prévention et le contrôle du tabagisme et du vapotage, en accordant un intérêt particulier aux jeunes. Dans cette optique, Promotion de la santé et prévention des maladies chroniques au Canada : recherche, politiques et pratiques sollicite des articles de recherche pertinents qui présentent des nouveaux résultats ou font la synthèse de données probantes existantes sur :

- des politiques, des interventions et des règlements en lien avec l'initiation au tabagisme ou au vapotage, les habitudes d'utilisation et de consommation, et l'abandon du tabagisme ou du vapotage, incluant les lacunes dans les politiques sur le tabagisme et le vapotage et les défis liés à leur mise en œuvre;

- les inégalités en santé liées à l’utilisation du tabac et des produits de vapotage et les méfaits qui y sont associés; et

- les associations entre l'utilisation de produits de vapotage et les comportements liés à l'abandon du tabagisme et à la réduction des méfaits, chez les fumeurs comme chez les non-fumeurs.

Les soumissions internationales seront prises en considération si elles contiennent des données ou des résultats canadiens (p. ex. dans le cadre d'une étude multipays) ou une analyse fondée sur des données probantes des implications pour la santé publique au Canada.

Veuillez consulter le site Web de la revue pour des renseignements sur les types d'articles et les lignes directrices à l'intention des auteurs. Prière de mentionner cet appel à contributions dans votre lettre d'accompagnement. Veuillez faire parvenir toutes vos soumissions et vos questions sur le processus de soumission ou sur la pertinence et la portée de manuscrits à PHAC.HPCDP.Journal-Revue .PSPMC.ASPC@canada.ca.

Échéance pour les soumissions : le 31 mars 2021.

\section{Références}

1. Santé Canada. Stratégie canadienne sur le tabac [Internet]. Ottawa (Ont.) : Santé Canada; [modifié le 10 janvier 2020; consultation le 20 novembre 2020]. En ligne à : https://www.canada.ca/fr/sante-canada/services/publications/vie-saine/strategie-tabac -canada.html

2. Santé Canada. Consultation sur l'avenir de la lutte contre le tabagisme au Canada : ce que nous avons entendu. Ottawa (Ont.) : Santé Canada; 2017. En ligne à : https://www.canada.ca/fr/sante-canada/services/publications/vie-saine/consultation-sur -lavenir-lutte-contre-tabagisme-ce-que-nous-avons-entendu.html

3. McDaniel PA, Smith EA, Malone RE. The tobacco endgame: a qualitative review and synthesis. Tob Control. 2016;25:594-604. https://doi.org/10.1136/tobaccocontrol-2015-052356

4. Le cabinet Tobacco Endgame. Atteindre un taux inférieur à 5 \% d'ici 2035 : rapport sur la lutte contre le tabagisme 2019. Ottawa (Ont.) : Association pulmonaire du Canada; 2019. En ligne à : https://www.poumon.ca/sites/default/files/EndGameReport-French_0.pdf

5. Santé Canada. Aperçu de la Stratégie canadienne sur le tabac. Ottawa (Ont.) : Santé Canada; 2018. En ligne à : https://www. canada.ca/fr/sante-canada/services/publications/vie-saine/strategie-tabac-canada/apercu-strategie-canadienne-tabac.html 